\title{
Clinical Study \\ Safety of Early Carotid Artery Stenting after Systemic Thrombolysis: A Single Center Experience
}

\author{
Fabrizio Sallustio, ${ }^{1,2}$ Giacomo Koch, ${ }^{1,2}$ Alessandro Rocco, ${ }^{1}$ Costanza Rossi, ${ }^{1}$ \\ Enrico Pampana, ${ }^{3}$ Roberto Gandini, ${ }^{3}$ Alessandro Meschini, ${ }^{3}$ Marina Diomedi, ${ }^{1,2}$ \\ Paolo Stanzione, ${ }^{1,2}$ and Silvia Di Legge ${ }^{1,2}$ \\ ${ }^{1}$ Department of Clinical Neurological Sciences, Stroke Unit, Policlinico Tor Vergata, Viale Oxford 81, 00133 Rome, Italy \\ ${ }^{2}$ I.R.C.C.S. Santa Lucia Foundation, Via Ardeatina 306, 00179 Rome, Italy \\ ${ }^{3}$ Department of Interventional Radiology, Policlinico Tor Vergata, Viale Oxford 81, 00133 Rome, Italy
}

Correspondence should be addressed to Silvia Di Legge, sdilegge@hotmail.com

Received 23 May 2011; Accepted 19 June 2011

Academic Editor: Arijana Lovrencic-Huzjan

Copyright ( $(2012$ Fabrizio Sallustio et al. This is an open access article distributed under the Creative Commons Attribution License, which permits unrestricted use, distribution, and reproduction in any medium, provided the original work is properly cited.

\begin{abstract}
Background. Patients with acute ischemic stroke due to internal carotid artery (ICA) disease are at high risk of early stroke recurrence. A combination of IV thrombolysis and early carotid artery stenting (CAS) may result in more effective secondary stroke prevention. Objective. We tested safety and durability of early CAS following IV thrombolysis in stroke patients with residual stenosis in the symptomatic ICA. Methods. Of consecutive patients treated with IV rtPA, those with residual ICA stenosis $\geq 70 \%$ or $<70 \%$ with an ulcerated plaque underwent early CAS ( $>24$ hours). The protocol included pre-rtPA MRI and MR angiography, and post-rtPA carotid ultrasound and CT angiography. Stroke severity was assessed by the NIH Stroke Scale (NIHSS). Three- and twelve-month stent patency was assessed by ultrasound. Twelve-month functional outcome was assessed by the modified Rankin Scale (mRS). Results. Of 145 consecutive IV rtPA-treated patients, 6 (4\%) underwent early CAS. Median age was 76 (range 67-78) years, median NIHSS at stroke onset was 12 (range 9-16) and 7 (range 7-8) before CAS. Median onset-to-CAS time was 48 (range 30-94) hours. A single self-expandable stent was implanted to cover the entire lesion in all patients. The procedure was uneventful in all patients. After 12 months, all patients had stent patency, and the functional outcome was favourable $(\mathrm{mRS} \leq 2)$ in all but 1 patient experiencing a recurrent stroke for new-onset atrial fibrillation. Conclusion. This small case series of a single centre suggests that early CAS may be considered a safe alternative to CEA after IV rtPA administration in selected patients at high risk of stroke recurrence.
\end{abstract}

\section{Introduction}

The incidence and management of early recurrent ischemic stroke after intravenous (IV) administration of recombinant tissue plasminogen activator (rtPA) have not been extensively investigated. Among stroke subtypes, patients with stroke due to large-artery atherosclerosis carry the highest risk of stroke recurrence, accounting for $37 \%$ of recurrences within 7 days [1]. A combination of IV thrombolysis and early carotid revascularization might result in more effective secondary stroke prevention strategy. However, at least in the first 24 hours after rtPA administration, the risk of intracranial haemorrhage associated with early reperfusion of a recently ischemic brain tissue might be increased $[2,3]$. Safety of early carotid endarterectomy (CEA) in patients with residual severe carotid stenosis following IV rtPA administration has been reported in small single-centre case series [4-6]. Emergency carotid artery stent (CAS) placement has safety comparable to that of emergent CEA [7] and may be indicated in selected patients with small infarct volume and mild neurological deficit [8]. Further, there is a substantial risk that a decrease in cerebral blood flow during emergency CEA in the affected hemisphere may turn the brain more vulnerable to ischemia. Conversely, early CAS might offer the advantage of treating a critical stenosis with a rare reduction in cerebral blood flow in the affected hemisphere during 
the intervention. However, the required double antiplatelet regimen that is administered before and after CAS procedure in order to prevent stent occlusion increases the potential risk of bleeding complications.

The aim of the present study was to analyze a cohort of patients undergoing early CAS after intravenous administration of rtPA for acute ischemic stroke. Safety of the procedure, patient outcome, and twelve-month durability of this approach were described.

\section{Methods}

Among consecutive patients treated with intravenous (IV) rtPA up to 4.5 hours [9], those with residual stenosis $\geq 70 \%$ or $<70 \%$ with an ulcerated plaque in the symptomatic internal carotid artery (ICA) underwent urgent CAS. All patients had (i) pre-rtPA treatment diffusion-weighted (DW) and perfusion-weighted (PW) MRI protocol and MR angiography (MRA) showing involvement of less than $1 / 3$ of middle cerebral artery (MCA) territory; (ii) head CT scan after rtPA administration and after CAS to rule out intracranial bleeding complications; (iii) post-rtPA treatment CT angiography (CTA) to identify the extent of residual ICA stenosis and features of plaque ulceration supporting an atherothrombotic origin of the stroke.

Age, sex, vascular risk factors, list of current medications, clinical and imaging data were collected for all patients. Stroke severity was assessed by the NIH Stroke Scale (NIHSS) at the following time points: before rtPA treatment, at the end of rtPA infusion, at 24 hours, at discharge, and 3 and 12 months after stroke onset. Severity of the stenosis was assessed by the NASCET criteria [10]. CAS was performed as soon as possible (but $>24$ hours) by an experienced team of interventional radiologists (EP, RG). No stent was placed within the first 24 hours after IV rtPA administration due to the absolute contraindication to start double antiplatelet treatment during this time frame. Prior to CAS procedure, ASA (100 mg/day) and clopidogrel (75 mg/day) were administered to all patients. During the procedure, intravenous heparin (5000 IU) was administered. To prevent bradycardia and hypotension during balloon inflation and stent deployment, prophylactic atropine $(0.5-1 \mathrm{mg})$ was administered in all patients 1 minute before stent deployment. Following the CAS procedure, ASA (100 mg/day) and clopidogrel (75 mg/day) were administered to all patients during 6 weeks, later only ASA (100 mg/day) was prescribed. All procedures were performed in an angiographic room; patients were assisted by an anaesthesiologist for invasive arterial pressure measurement and oximetry monitoring. The patients, under light sedation, were kept conscious. The procedure adopted in our institution has been previously described [11]. A single self-expandable stent was implanted to cover the entire lesion in all patients. The stent used was the Cristallo Ideale (Carotid Self-Expanding Stent System; Medtronic) and the WALLSTENT Monorail Endoprosthesis (Boston Scientific, US). Protection devices were the EPI Filter Wire EZ Embolic Protection System (Boston Scientific Corporation, USA) or The SPIDER Embolic Protection Device (ev3, Plymouth, $\mathrm{MN}$ ). All stent were postdilated with noncompliant balloons
TABle 1: Demographic and clinical data of six patients who had early carotid artery stenting after thrombolysis.

\begin{tabular}{lc}
\hline & No. of patients* \\
\hline Age, years & $76(67-78)$ \\
Females, $n$ (proportion) & $2(0.33)$ \\
Onset-to-CAS time, days & $2.5(2-4)$ \\
Onset-to-CAS time, hours & $48(30-94)$ \\
NIHSS onset (pre-rtPA) & $12(9-16)$ \\
NIHSS score before CAS & $7(7-8)$ \\
Right ICA involvement/ $n$ (proportion) & $4(0.67)$ \\
\hline Vascular risk factors: & \\
$\quad$ Smoking & 2 \\
$\quad$ Hypertension & 6 \\
$\quad$ Diabetes & 1 \\
$\quad$ Previous TIA/stroke & 2 \\
$\quad$ Hypercholesterolemia & 3 \\
\hline 12-month NIHSS score & $2.5(2-5)$ \\
\hline Numbers are medians $( \pm$ IQR); CAS: carotid artery stenting; NIHSS: \\
National Institute of Health Stroke Scale; TIA: transient ischemic attack.
\end{tabular}

(range 5-6 mm). In all patients, after stent deployment, a postprocedural angiography was performed to evaluate the eventual residual stenosis and intracranial circulation.

After the procedure, patients were admitted to the stroke unit and managed according to the guidelines. Three-month and twelve-month stent patency was assessed by ultrasound. The rate of recurrent cerebrovascular events was assessed at 3, 6 and 12 months. Twelve-month followup included assessment for stroke severity by the NIHSS and functional outcome by the modified Rankin Scale (mRS).

\section{Results}

Since October 2006, 145 patients received IV rtPA within 4.5 hours after the onset of symptoms of acute ischemic stroke in our Institution. Within this group, subsequent CAS was performed in 6/145 (4\%) patients with evidence of residual stenosis $\geq 70 \%$ or $<70 \%$ with ulcerated plaque in the symptomatic ICA associated with unstable or ulcerated plaque, as detected by post-rtPA CTA. They were 4 males and 2 females, median age (IQR) was 76 (67-78) years, median admission NIHSS (IQR) score was 12 (9-16), and median (IQR) onset-to-IV rtPA administration was 175 (120-240) minutes. Median (IQR) 24-hour NIHSS change was -3 (-9;-2). The median NIHSS (IQR) score before CAS was 7 (7-8). Median (IQR) onset-to-CAS time was 48 (30-94) hours. Median (range) pretreatment DWI lesion volume was $26 \mathrm{~cm}^{3}$ (652 ), and median (range) DWI lesion volume on follow-up examination (days 5-7 after stroke onset) was $31 \mathrm{~cm}^{3}$ (5-57). The procedure was uneventful in all patients. Demographic, clinical, and outcome data are shown in Tables 1 and 2. On pre-rtPA treatment MRA, an intracranial occlusion was detected in 3 patients (Table 2). On CTA performed before CAS, there was a persisting MCA steno/occlusive disease in one patient only. However, angiographic study performed at the end on the CAS procedure yielded normal findings 


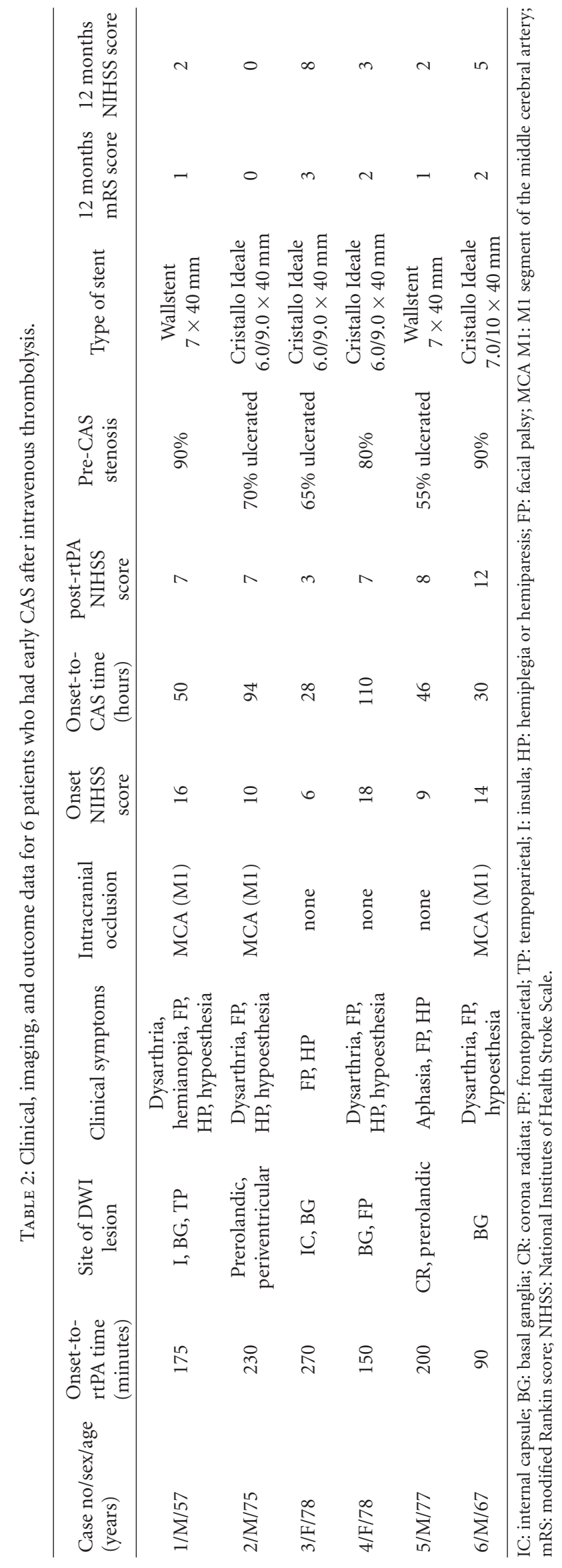


in all patients. There were no bleeding complications on posttreatment CT scan, and all patients were discharged on daily ASA (100 mg/day) plus clopidogrel ( $75 \mathrm{mg} /$ day). Regarding the discharge time, the two subgroups of rtPAtreated stroke patients in whom the early CAS was performed (mean \pm SD $5.6 \pm 1.8$ days) and rtPA-treated stroke patients without the CAS procedure (mean \pm SD $6.8 \pm 2.6$ days) did not differ in mean discharge time $(P=0.29)$.

All stents were patent at 3-month and 12-month ultrasound examination. Twelve-month functional outcome was favourable $(\mathrm{mRS} \leq 2)$ in all but 1 patient experiencing a recurrent stroke after 4 months for new-onset atrial fibrillation.

\section{Discussion}

This small case series suggests that early treatment with CAS is both feasible and safe in selected patients previously treated with IV rtPA for an acute ischemic stroke. All stents were patent at 3- and 12-month follow-up evaluation. The outcome at 12 months was favourable in all but one patient experiencing a recurrent stroke four months following the index event associated with a new-onset paroxysmal atrial fibrillation.

A major concern about the safety of this approach is that previous systemic thrombolysis might increase the risk of hemorrhagic transformation thus making early reperfusion procedures potentially dangerous [2, 3]. For this reason, CAS was performed at least 24 hours after IV rtPA administration (with a median onset-to-CAS time of 2.5 days) when the hemocoagulative effects of thrombolytic therapy decline toward baseline [12]. Further, additional risk of hemorrhagic transformation could be determined by the need of administer double antiplatelet regimen to prevent in-stent restenosis. Feasibility and safety of urgent CAS after stroke have been previously investigated. In the study of Zaidat et al. [8], clinical and radiological data on a total of 38 patients with 39 procedures were reviewed. The median initial NIHSS score was 8 . The carotid artery showed severe to high-grade stenosis in 28 patients, dissection was present in 6 , and the rest $(n=4)$ had an acute occlusion treated with thrombolysis followed by CAS. The mean time from stroke onset to CAS was $55( \pm 34)$ hours. Complete recanalization was achieved in 95\% procedures. Neurological deterioration occurred after three procedures $(8 \%)$, with minor nondisabling stroke in two and death from intracranial hemorrhage in one. The authors concluded that early CAS seems to be safe after acute ischemic stroke if infarction volume is small and neurological deficit is mild. In a single-centre Italian registry [13], early treatment with protected carotid stenting was both feasible and safe in 43 selected patients with TIA or minor stroke (with cerebral ischemic lesion smaller than $2.5 \mathrm{~cm}$ ). Patients who had a TIA underwent urgent CAS within 24 hours of the cerebral event, while patients who had had a minor stroke underwent deferred CAS (mean time, 6.5 days; range, 2-28 days). Similar to our findings, an urgent endovascular approach was associated with a satisfactory outcome considering the very high-risk profile of the patient population. However, the novelty of our study, compared to earlier ones, is that we have focused our attention on patients who had received IV rtPA before being submitted to a CAS procedure. Conversely, in one study [8], only a minority of patients $(n=4)$ had received IV rtPA before CAS. On the other hand, safety of early CEA following intravenous thrombolysis has been described in small singlecentre case series of patients with an acute ischemic stroke. In the first study [4] five patients underwent early CEA (range 6-45 hours) after rtPA administration. The procedure was safe, and the outcome was favourable in all patients with no recurrent stroke (follow-up of 5-22 months). In the study of Bartoli et al. [5] early CEA was performed with a median of 8 days (range 1-16 days) after rtPA treatment in patients with median NIHSS of 12 (range 5-21). Also in this case series $(n=12)$ full vessel patency was achieved in all patients, and 3-month outcome was favourable in 10/12 (83\%) patients. In the study of Crozier et al. [6] only $10 / 450$ (2\%) of patients treated with IV rtPA were eligible for early CEA; surgery was performed with a median of 8 (range 2-23) days after the index event. The procedure was safe, and the clinical outcome (up to six weeks) was favourable in all patients. Taken together, the results of these studies suggest that first, CEA can be safely performed shortly after IV rtPA administration; second, only a small percentage of patients treated with IV rtPA are appropriate candidates for reperfusion treatment; third, the best time to perform the procedure remains to be determined. Based on our results, similar considerations can apply to CAS after thrombolysis.

Although not as safe as elective carotid stent placement, early CAS performed with a mean time from stroke onset of $55( \pm 34)$ hours seems to be safe after an acute ischemic stroke if infarction volume is small and neurological deficit is mild [8]. Further, emergency carotid stent placement has safety comparable to that of emergent CEA, sharing similar risks of hemorrhagic transformation related to early reperfusion [7]. A major concern might be in the increased risk of hemorrhagic transformation related to the double antiplatelet treatment required after CAS. However, we did not observe any hemorrhagic complications, and none of the patients presented clinical deterioration after the procedure.

In conclusion, this is the first paper evaluating safety, durability, and patients outcome of early CAS after systemic IV rtPA administration. This small case series of a single centre suggests that CAS may be considered a safe alternative to CEA after rtPA administration in preventing early stroke recurrence in selected patients. These results apply to patients with residual significant ICA stenosis or ulcerated plaque after IV thrombolysis and pretreatment DWI lesion volume not exceeding $1 / 3$ of the MCA territory.

\section{References}

[1] J. K. Lovett, A. J. Coull, and P. M. Rothwell, "Early risk of recurrence by subtype of ischemic stroke in population-based incidence studies," Neurology, vol. 62, no. 4, pp. 569-573, 2004.

[2] Y. F. Wang, S. E. Tsirka, S. Strickland, P. E. Stieg, S. G. Soriano, and S. A. Lipton, "Tissue plasminogen activator (tPA) increases neuronal damage after focal cerebral ischemia in 
wild-type and tPA-deficient mice," Nature Medicine, vol. 4, no. 2, pp. 228-231, 1998.

[3] M. Ning, K. L. Furie, W. J. Koroshetz et al., "Association between tPA therapy and raised early matrix metalloproteinase-9 in acute stroke," Neurology, vol. 66, no. 10, pp. 1550-1555, 2006.

[4] C. M. McPherson, D. Woo, P. L. Cohen et al., "Early carotid endarterectomy for critical carotid artery stenosis after thrombolysis therapy in acute ischemic stroke in the middle cerebral artery," Stroke, vol. 32, no. 9, pp. 2075-2079, 2001.

[5] M. A. Bartoli, C. Squarcioni, F. Nicoli et al., "Early carotid endarterectomy after intravenous thrombolysis for acute ischaemic stroke," European Journal of Vascular and Endovascular Surgery, vol. 37, no. 5, pp. 512-518, 2009.

[6] J. E. M. Crozier, J. Reid, G. H. Welch, K. W. Muir, and W. P. Stuart, "Early carotid endarterectomy following thrombolysis in the hyperacute treatment of stroke," British Journal of Surgery, vol. 98, no. 2, pp. 235-238, 2011.

[7] K. Imai, T. Mori, H. Izumoto, M. Watanabe, and K. Majima, "Emergency carotid artery stent placement in patients with acute ischemic stroke," American Journal of Neuroradiology, vol. 26, no. 5, pp. 1249-1258, 2005.

[8] O. O. Zaidat, M. J. Alexander, J. I. Suarez et al., "Early carotid artery stenting and angioplasty in patients with acute ischemic stroke," Neurosurgery, vol. 55, no. 6, pp. 1237-1242, 2004.

[9] Stroke Prevention and Educational Awareness Diffusion (SPREAD), 6th Edition, 2010, http://www.spread.it/.

[10] D. W. Taylor and H. J. M. Barnett, "Beneficial effect of carotid endarterectomy in symptomatic patients with highgrade carotid stenosis," The New England Journal of Medicine, vol. 325, no. 7, pp. 445-453, 1991.

[11] G. Simonetti, R. Gandini, F. Versaci et al., "Carotid artery stenting: a single-centre experience with up to 8 years' followup," European Radiology, vol. 19, no. 4, pp. 982-989, 2009.

[12] K. Fassbender, C. E. Dempfle, O. Mielke et al., "Changes in coagulation and fibrinolysis markers in acute ischemic stroke treated with recombinant tissue plasminogen activator," Stroke, vol. 30, no. 10, pp. 2101-2104, 1999.

[13] C. Setacci, G. de Donato, E. Chisci, and F. Setacci, "Carotid artery stenting in recently symptomatic patients: a single center experience," Annals of Vascular Surgery, vol. 24, no. 4, pp. 474-479, 2010. 


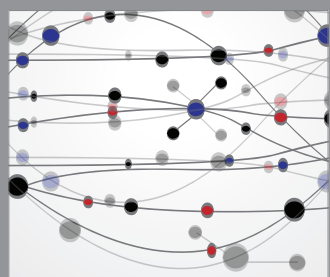

The Scientific World Journal
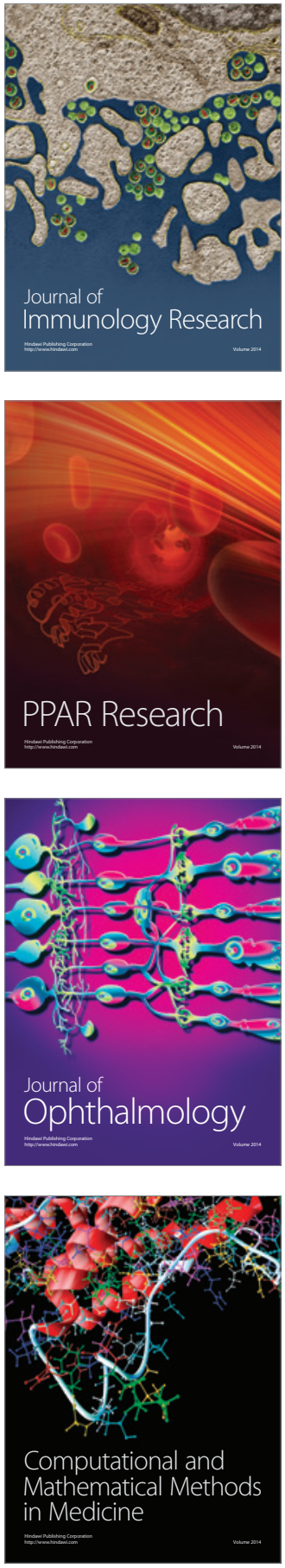

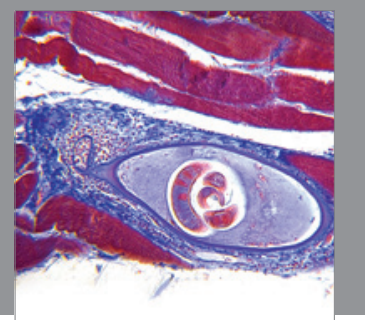

Gastroenterology

Research and Practice
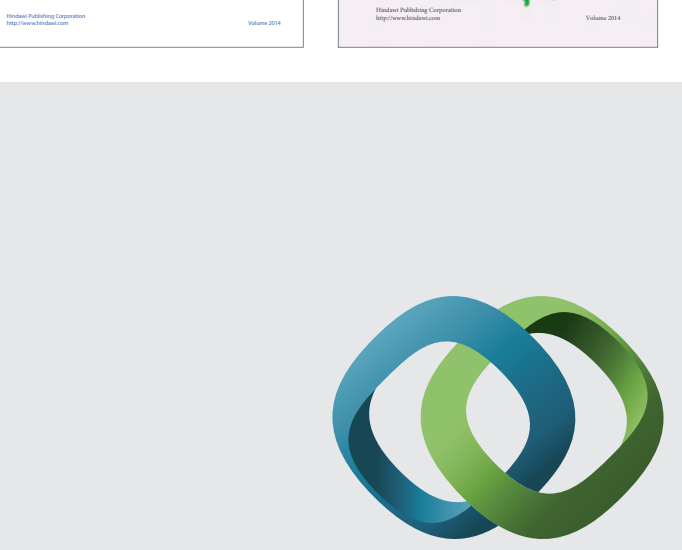

\section{Hindawi}

Submit your manuscripts at

http://www.hindawi.com
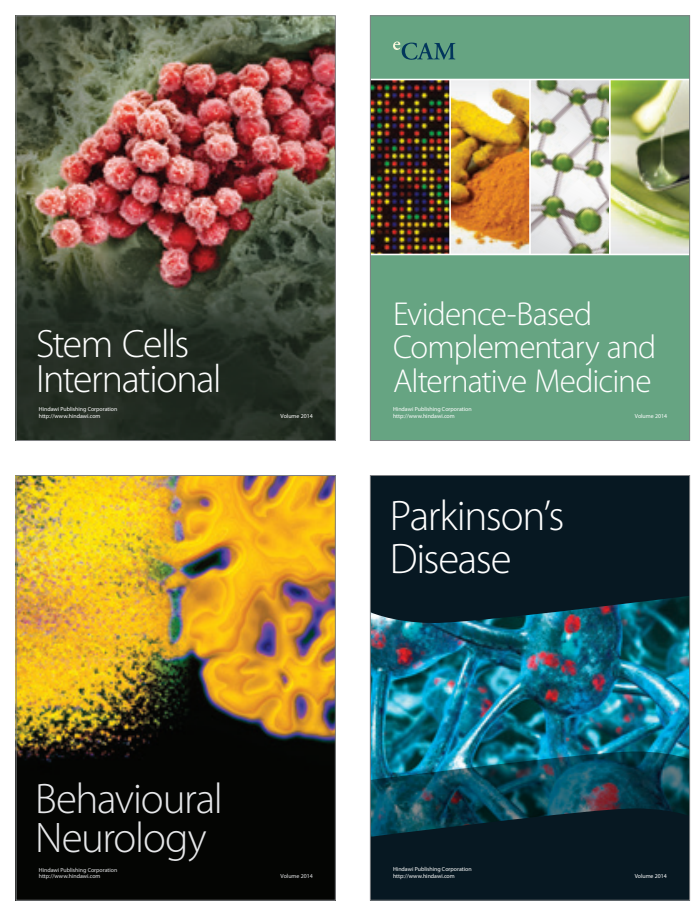

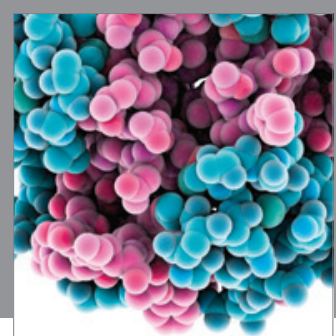

Journal of
Diabetes Research

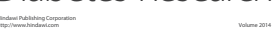

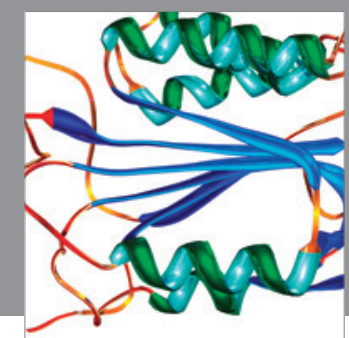

Disease Markers
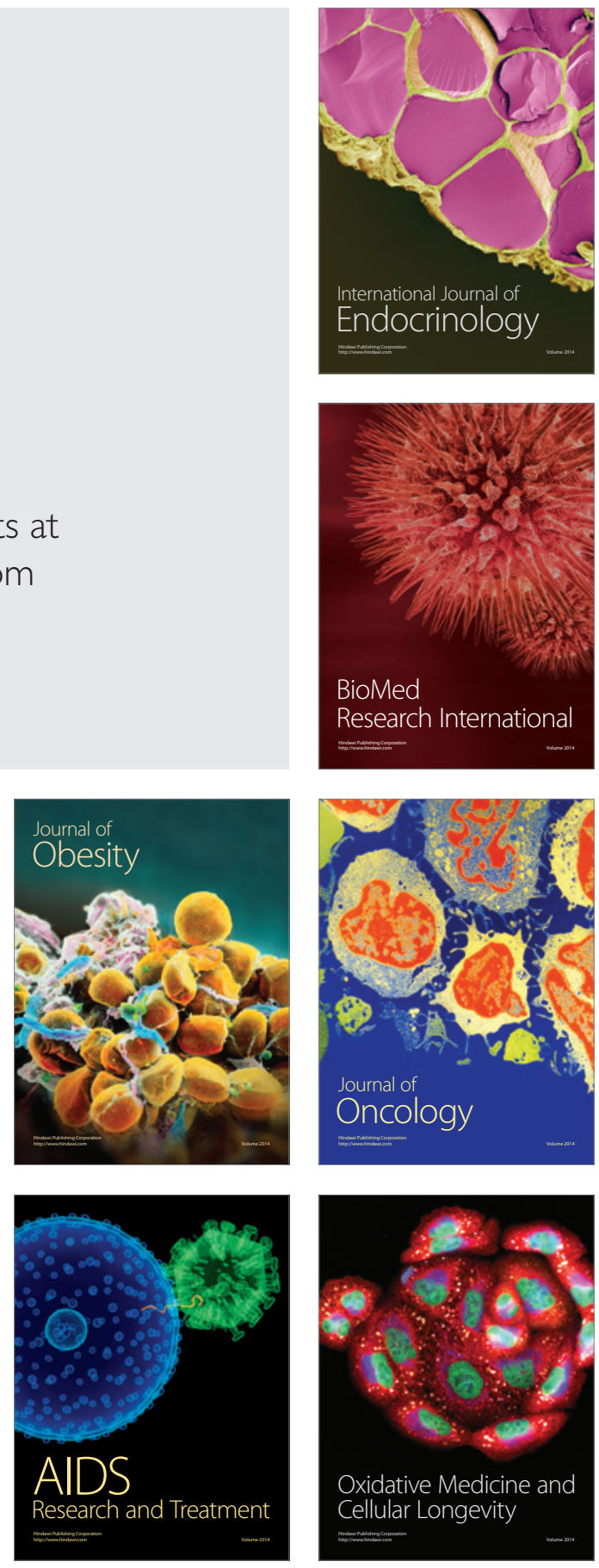